\title{
Outcomes and Treatment of Acute Hepatitis C Virus Infection in a United States Population
}

\author{
Kathleen Corey ${ }^{1, \wedge}$, Andrew S. Ross $2,{ }^{\wedge},{ }^{\star}$, Alysse Wurcel $2,{ }^{* *}$, Julian Schulze zur Wiesch ${ }^{3}$, \\ Arthur Y. Kim ${ }^{3}$, Georg M. Lauer ${ }^{3}$, and Raymond T. Chung ${ }^{2}$ \\ 1 Medical Services, Massachusetts General Hospital, Harvard Medical School, Boston, MA 02114 \\ 2 Gastrointestinal Unit, Massachusetts General Hospital, Harvard Medical School, Boston, MA 02114 \\ 3 Partners AIDS Research Center, Massachusetts General Hospital, Harvard Medical School, Boston, MA \\ 02114
}

\section{Abstract}

Introduction- Acute hepatitis $\mathrm{C}$ infection progresses to chronic infection in up to $80 \%$ of infected persons. Reports from Europe indicate that early treatment of acute hepatitis $\mathrm{C}$ infection produces sustained virologic response rates as high as $80-98 \%$. However, the outcome of acute hepatitis C infection in United States cohorts is not well characterized.

Methods-We describe the clinical course of 28 episodes of acute hepatitis $C$ infection in 24 persons at our institution.

Results- Of the 28 infections, seven episodes resolved spontaneously. Of the remaining 21 episodes, 16 were treated, while five did not receive treatment. Of the 16 treated episodes, 4 received interferon and ribavirin, 11 received pegylated interferon and ribavirin, and one was treated initially with interferon monotherapy followed by PEG monotherapy. Among those episodes treated with interferon, 3 of 4 experienced sustained virologic response (SVR). Among those episodes treated with pegylated interferon, all 12 achieved SVR. In total, 15 of 16 treated patients (94\%) experienced SVR. In all, 18 of the 24 patients (75\%) experienced spontaneous or treatment-induced sustained virologic clearance.

Conclusion-Our experience with treated and untreated acute HCV infection is comparable to that observed in Europe. Patients treated with antiviral therapy had an excellent response. Randomized trials to investigate immediate versus delayed treatment of acute hepatitis $\mathrm{C}$ infection are warranted. In view of these strongly positive outcomes, increased vigilance for acute hepatitis $\mathrm{C}$ becomes essential.

\section{INTRODUCTION}

Recent estimates have indicated that up to two percent of the population in the United States (US) is infected with hepatitis $\mathrm{C}$ virus (HCV) (1). Chronic HCV infection is the most common

Address correspondence to: Raymond T. Chung, M.D., Gastrointestinal Unit, GRJ-825, Massachusetts General Hospital, Boston, MA 02114, Phone 617-724-7562, Fax 617-726-5895, Email: rtchung @ partners.org.

${ }^{\wedge}$ These authors contributed equally to this manuscript.

* Present address: University of Chicago, GI Section, Chicago, IL

** Present address: University of Pennsylvania School of Medicine, Philadelphia, PA

Publisher's Disclaimer: This is a PDF file of an unedited manuscript that has been accepted for publication. As a service to our customers we are providing this early version of the manuscript. The manuscript will undergo copyediting, typesetting, and review of the resulting proof before it is published in its final citable form. Please note that during the production process errors may be discovered which could affect the content, and all legal disclaimers that apply to the journal pertain. 
cause of chronic hepatitis worldwide and cirrhosis attributable to HCV infection is the leading indication for liver transplantation.

While the prevalence of chronic $\mathrm{HCV}$ infection is of epidemic proportion, the incidence of acute HCV infection has declined over the past 20 years. This decrease has been attributable to the successful screening of blood products for $\mathrm{HCV}$, decreases in injection drug use and improved aseptic technique. Despite these advances, an estimated 40,000 cases of acute HCV occur annually in the United States (2).

Untreated, acute HCV infection progresses to chronic infection in 50-80\% of patients (3-5) while the remainder appear to be able to clear the virus on their own. Several studies have suggested that female sex, symptomatic disease and high peak bilirubin levels are the strongest predictors of spontaneous viral clearance(6). Data with regard to antiviral therapy for acute $\mathrm{HCV}$ infection have been difficult to interpret, primarily due to small sample sizes and lack of randomization. A 1999 meta-analysis found that treatment with interferon alfa-2b (IFN) at doses of at least three MIU thrice weekly for a minimum of 12 weeks was superior to no treatment in terms of normalization of transaminases and sustained virologic response (SVR) rates (6).

A recent study from Germany observed that 43 of 44 patients (98\%) with acute HCV treated with five million units of interferon daily for four weeks followed by five MIU thrice weekly for 20 weeks experienced SVR (4). Another report described SVR in $90 \%$ of patients treated with IFN alone or in combination with ribavirin (RBV) (5).

Gerlach and colleagues (6) recently observed that in a cohort of 60 patients presenting with acute $\mathrm{HCV}$ infection, $52 \%$ of symptomatic patients and $0 \%$ of asymptomatic patients achieved spontaneous viral clearance. In addition, among those who remained viremic, initiation of antiviral therapy with IFN monotherapy 12 weeks following the onset of acute hepatitis produced an SVR rate of $80 \%$. (6)

European studies describing the treatment of chronic HCV infection usually report higher rates of SVR than do studies performed in North America. This has been attributed in part to the higher prevalence of the more treatment-responsive viral genotypes 2 and 3 in Europe. To date, there have been no reports describing the natural history and treatment of acute HCV infection in the United States. Here we describe the clinical course of 24 patients with 28 episodes of acute $\mathrm{HCV}$ diagnosed at our institution.

\section{METHODS}

\section{Patients}

The clinic charts and electronic medical records of patients diagnosed with acute HCV during the period from 1998-2005 were reviewed. The following data were recorded: age, sex, probable exposure date and type of exposure, $\mathrm{HCV}$ infection risk factors, $\mathrm{HIV}$ infection status, symptoms, peak ALT, peak bilirubin, the presence of antibody to HCV, HCV genotype, HCV RNA, antiviral therapy, doses, duration and complications. In some patients in whom viremic sera were not available for genotyping, serotyping for $\mathrm{HCV}$-genotype was performed using the Murex HCV Serotyping 1-6 assay (Abbott, Wiesbaden, Germany) according to the manufacturer's instructions. Informed consent was obtained from all patients, and institutional review board approval was obtained. Patients were excluded if they had incomplete charts.

\section{Acute HCV Infection}

The diagnosis of acute HCV infection was made if a patient presented with (1) either a documented anti-HCV seroconversion with positive HCV RNA; (2) a positive anti-HCV with 
detectable HCV RNA and an ALT elevated to greater than 10x the upper limit of normal and a history of recent high risk exposure; or (3) a negative anti-HCV with a positive HCV RNA.

\section{Antiviral Therapy}

At the clinician's discretion, patients were treated with standard IFN-alfa- $2 b$ at doses between 9-42 MIU weekly or pegylated interferon alfa-2a (PEG) at a dose of $180 \mathrm{mcg}$ weekly. In addition, most patients received combination therapy with RBV at doses of 1000-1200 mg daily based on body weight less than or greater than $75 \mathrm{~kg}$. The duration of therapy varied by individual patient.

\section{Virologic Response}

Patients were classified as having an end of treatment virologic response (ETVR) if they had an undetectable $\mathrm{HCV}$ viral load by qualitative assay at the end of treatment. A sustained virologic response (SVR) was defined as an undetectable HCV RNA by qualitative assay 24 weeks following the cessation of any course of therapy.

\section{RESULTS}

\section{Patients and Exposure (Table 1)}

Twenty-four patients $(15 \mathrm{~F} / 9 \mathrm{M})$ were diagnosed with a total of 28 discrete episodes of acute HCV between February 1998 and June 2005. None of the patients were excluded for incomplete records. The patients ranged in age from 15 to 63 years (mean $34+/-13$ ). Risk exposures for $\mathrm{HCV}$ infection included: injection drug use (IDU) in 13 patients, needle-stick injuries in five patients, blood transfusions in one patient, shared razors in one patient, and unprotected sex in one patient. Three patients who were diagnosed with acute HCV had no clearly identifiable exposure. The median duration of follow-up was 90 weeks (range 10-187 weeks).

\section{Signs and Symptoms}

The following were reported as initial symptoms: fatigue $(n=5)$, fever $(n=4)$, nausea $(n=8)$, abdominal pain $(\mathrm{n}=3)$, rash $(\mathrm{n}=1)$, and myalgias $(\mathrm{n}=1)$. Six patients reported jaundice as the initial sign of $\mathrm{HCV}$ infection; two patients were completely asymptomatic.

\section{Liver Tests, Viral Load and Genotype}

All 24 patients reviewed had abnormal serum ALT levels at initial presentation. Four women had discrete marked ALT elevations after exposures on two separate occasions, resulting in a total of 28 discrete acute infections. The mean peak ALT was $1649(+/-1454) \mathrm{U} / \mathrm{L}$. The total bilirubin was elevated in $17(60 \%)$ patients with a mean peak total bilirubin of $4.4(+/-4.6 \mathrm{mg} /$ dl). The mean initial HCV RNA was 325,590 (+/- 453,534) IU/mL (ref range <600$700,000 \mathrm{IU} / \mathrm{mL}$ ) with a median value of $63149+/-15787 \mathrm{IU} / \mathrm{mL}$. Viral genotype or serotype was identified in 27 (96\%) of the 28 infections. Twenty-three (82\%) infections were genotype 1 , four (14\%) were genotype 2 or 3 , and one was neither genotypable nor serotypable. Of note, all four patients with multiple episodes had infections with discordant subtypes (1a and 1b).

\section{Disease Course and Treatment}

Of the 24 patients, five $(20 \%$; 4F, $1 \mathrm{M})$ cleared seven episodes of $\mathrm{HCV}$ infection spontaneously (Figure 1). Three of these presented twice with symptoms of acute hepatitis after discrete exposures. Of these patients, two spontaneously cleared viremia both times; the third was treated with PEG-IFN after the first episode and spontaneously cleared the second episode. All five were injection drug users and had elevated bilirubin levels and had ALT levels greater than $1000 \mathrm{U} / \mathrm{L}$ at presentation. Of the seven acute episodes with HCV that cleared spontaneously, six were genotype 1 and one was unknown. For those patients who were not 
treated immediately and who experienced spontaneous clearance, time from the onset of symptoms to viral clearance ranged from 10 to 96 days (median 49 days).

Fifteen patients (62.5\%) received antiviral therapy for 16 episodes of acute $\mathrm{HCV}$ infection. One patient (\#24) experienced two discrete acute infections after documented exposure to IDU and was treated twice. Initial therapy was with IFN $+/-$ RBV in four patients and PEG-IFN +/ - RBV in 11 patients. One patient (patient \#9) who relapsed after treatment with IFN monotherapy (patient was on hemodialysis) was subsequently treated with PEG-IFN monotherapy. Therapy was initiated at a median of 21 days (range 14-183) after symptoms were reported. The mean weekly dose (where known) of IFN was $26(+/-5.9)$ MIU and the mean cumulative dose of IFN was $1011.25(+/-206.6)$ MIU. The mean weekly dose of PEGIFN $180 \mathrm{mcg}$ and the mean cumulative dose of PEG-INF was $3600 \mathrm{mcg}$. The mean daily dose of RBV was $944(+/-87.5) \mathrm{mg}$. The mean duration of therapy was $30.7+/-12$ weeks.

All 15 treated patients had an end of treatment virologic response. The mean week 12 $\log _{10} \mathrm{HCV}$ RNA decline was $4.79(+/-1.23)$ in treated patients. All patients cleared HCV RNA by week 12. Two patients who had ETVR experienced a relapse of HCV infection. Of these, one patient (\#7) is currently viremic and has elected not to be retreated. The other patient (\#9) was on hemodialysis, relapsed after receiving standard IFN, and successfully achieved SVR with PEG-IFN monotherapy. Of note, this patient then underwent a living related kidney transplant complicated by hyperacute rejection and OKT3 treatment. Despite this, she has remained HCV RNA negative for four years. All patients have completed treatment and all achieved ETVR. Overall, 15 of the 16 episodes (94\%) resulted in sustained virologic response (SVR).

Taken together, of the 28 acute $\mathrm{HCV}$ episodes, 22 resulted in spontaneous or treatment-induced sustained virologic response, and 6 resulted in persistent viremia. Of these 6 viremic patients, 5 were not treated. All 5 had absolute or relative contraindications to therapy (three active drug users and two with severe depression). Of the 24 patients in all, 18 (75\%) experienced sustained virologic clearance, and 6 remain viremic.

\section{DISCUSSION}

Here we describe the clinical course of the first and largest series of 24 American patients diagnosed with acute $\mathrm{HCV}$ infection. In our series, five of the patients cleared the virus spontaneously on seven occasions; all five of these patients were symptomatic with elevated transaminases and jaundice. Fifteen patients (comprising 16 episodes) completed antiviral therapy; all experienced initial virologic clearance, and all but one (93\% of patients, $94 \%$ of episodes) experienced sustained virologic response. Of the original 24 patients with acute $\mathrm{HCV}, 6$ remain viremic. However, 5 of these 6 were not offered therapy because of contraindications. Therefore, in factoring "as treated" or "real world" success rates, sustained virologic clearance (spontaneous or treatment-induced) was ultimately observed in 18 of 24 patients $(75 \%)$, still a very favorable outcome.

Gerlach and colleagues (6) have demonstrated that the majority of patients who spontaneously clear hepatitis $\mathrm{C}$ virus do so within three months of the onset of symptoms. These authors found that treatment of persistently viremic patients after three months is associated with very high rates of SVR. Among patients who did not receive immediate treatment, our findings were consistent with the reports by Gerlach et al. (6) in that the median time from symptom onset to spontaneous viral clearance was 49 days with a range from 10 to 96 days. Antiviral therapy in our patients was initiated at a median of 21 days (range 14-183 days) after the onset of symptoms. It is unclear whether patients who were treated early might otherwise have cleared 
spontaneously. Treatment strategy studies should address whether delayed initiation of treatment should be undertaken, particularly in symptomatic patients.

Our study included four patients who each presented with two distinct episodes of acute hepatitis $\mathrm{C}$ infection. We believe that in each patient the presentations represented two distinct infections rather than a single infection reemerging after a period of quiescence. This is supported by the finding of different viral subtypes in the primary and secondary presentation and the identification of a high-risk behavior in each patient prior to each presentation. Mehta et al (7) have recently reported on the incidence of HCV in injection drug users both naïve and previously exposed to acute HCV. They followed 164 patients not previously exposed to HCV and 98 patients previously exposed. While the occurrence of viremia was not significantly different in the two sets of patients, in the subset of HIV negative patients the incidence of viremia was significantly lower in the previously exposed patients than in the HCV naïve patients. In addition, $\mathrm{HCV}$-exposed patients who were re-infected with HCV had notably lower $\mathrm{HCV}$ viral loads than their naïve counterparts and were 12 times less likely to develop chronic $\mathrm{HCV}$ when compared to their HCV naïve counterparts These findings are in alignment with the findings in our study, in which two of four patients with multiple episodes of acute HCV cleared both infections spontaneously and a third (\#18) who did not initially clear spontaneously and was treated successfully, spontaneously cleared $\mathrm{HCV}$ after reinfection. The fourth patient had comparable peak HCV RNA levels after each acute infection.

There is little data regarding the natural history of acute HCV infection. Reports regarding the outcome and the treatment of acute infection have been confined to European studies, where two large cohorts of acute HCV infection have had excellent success in achieving SVR. In addition, several recent European studies have demonstrated an increased incidence in acute hepatitis $\mathrm{C}$ infection in men having sex with men $(8,9)$. Still, in chronic HCV infection clinical trials European populations have generally had higher SVR rates when compared to US patients. The reasons for these higher response rates are unknown but may include differences in BMI. We therefore sought to characterize the outcome of acute $\mathrm{HCV}$ and its treatment in a cohort of US patients who were infected predominantly with HCV genotype 1. We found that the use of interferon or peginterferon alone or in combination with RBV for up to 24 weeks after presentation produced SVR in all but one of our patients with acute HCV.

While our study is unique in undertaking the evaluation of acute HCV in a US cohort it may be limited by its retrospective nature and confinement to a tertiary referral center. It is therefore possible that these findings may not be generalizable to the US population at large. However, we believe this study highlights the importance of diagnosing and successfully managing acute $\mathrm{HCV}$ in American patients.

In summary, the superb success in achieving SVR in this US cohort of acute HCV mirrors that observed in Europe and emphasizes the importance of prompt recognition of acute HCV infection. The optimal duration of therapy for acute HCV still requires clarification. While patients in this cohort who were treated received therapy early, the finding of spontaneous clearance among untreated patients at up to 12 weeks form onset of symptoms suggests that a watch and treat approach can be effective in this condition.

\section{References}

1. Alter MJ, Kruszon-Moran D, Nainan OV, et al. The prevalence of hepatitis virus infection in the United States, 1988 through 1994. N Engl J Med 1999;341:556-62. [PubMed: 10451460]

2. Armstrong GL, Alter MJ, McQuillan GM, Margolis HS. The past incidence of hepatitis C virus infection: implications for the future burden of chronic liver disease in the United States. Hepatology 2000;31:777-82. [PubMed: 10706572] 
3. Hofer H, Watkins-Riedel T, Janata O, et al. Spontaneous viral clearance in patients with acute hepatitis C can be predicted by repeated measurements of serum viral load. Hepatology 2003;37:60-64. [PubMed: 12500189]

4. Jaeckel E, Cornberg M, Wedemeyer $\mathrm{H}$, et al. Treatment of acute hepatitis $\mathrm{C}$ with interferon alfa- $2 \mathrm{~b}$. N Engl J Med 2001;345:1452-1457. [PubMed: 11794193]

5. Larghi A, Zuin M, Crosignani A, et al. Outcome of an outbreak of acute hepatitis $\mathrm{C}$ among healthy volunteers participating in pharmacokinetics studies. Hepatology 2002;35:993-1000. [PubMed: 11981749]

6. Gerlach JT, Diepolder HM, Zachoval R, et al. Acute hepatitis C: high rate of both spontaneous and treatment-induced viral clearance. Gastroenterology 2003;125:80-85. [PubMed: 12851873]

7. Mehta SH, Cox A, Hoover D, et al. Protection against persistence of hepatitis C. Lancet 2002;359:1478-83. [PubMed: 11988247]

8. Rauch, A.; Rickenbach, M.; Weber, R. Association of unsafe sex and increased incidence of hepatitis $\mathrm{C}$ infection in HIV-infected men who have sex with men; 12th Conference on Retroviruses and Opportunistic Infections 2005; Abstract \#941.

9. Chaix, MI.; Serpaggi, J.; Batisse, D., et al. Homosexually transmitted HCV acute infection related to a cluster genotype-4 HCV in HIV-1 infected men and inefficacy of early anti-viral therapy; 12th Conference on Retroviruses and Opportunistic Infections 2005; Abstract \#122

\section{Abbreviations}

$\mathrm{HCV}$

hepatitis $\mathrm{C}$ virus

IFN

interferon alfa- $2 \mathrm{a} / 2 \mathrm{~b}$

PEG-IFN

pegylated interferon alfa- $2 \mathrm{a} / 2 \mathrm{~b}$

RBV

ribavirin

SVR

sustained virologic response

ETVR

end of treatment virologic response 


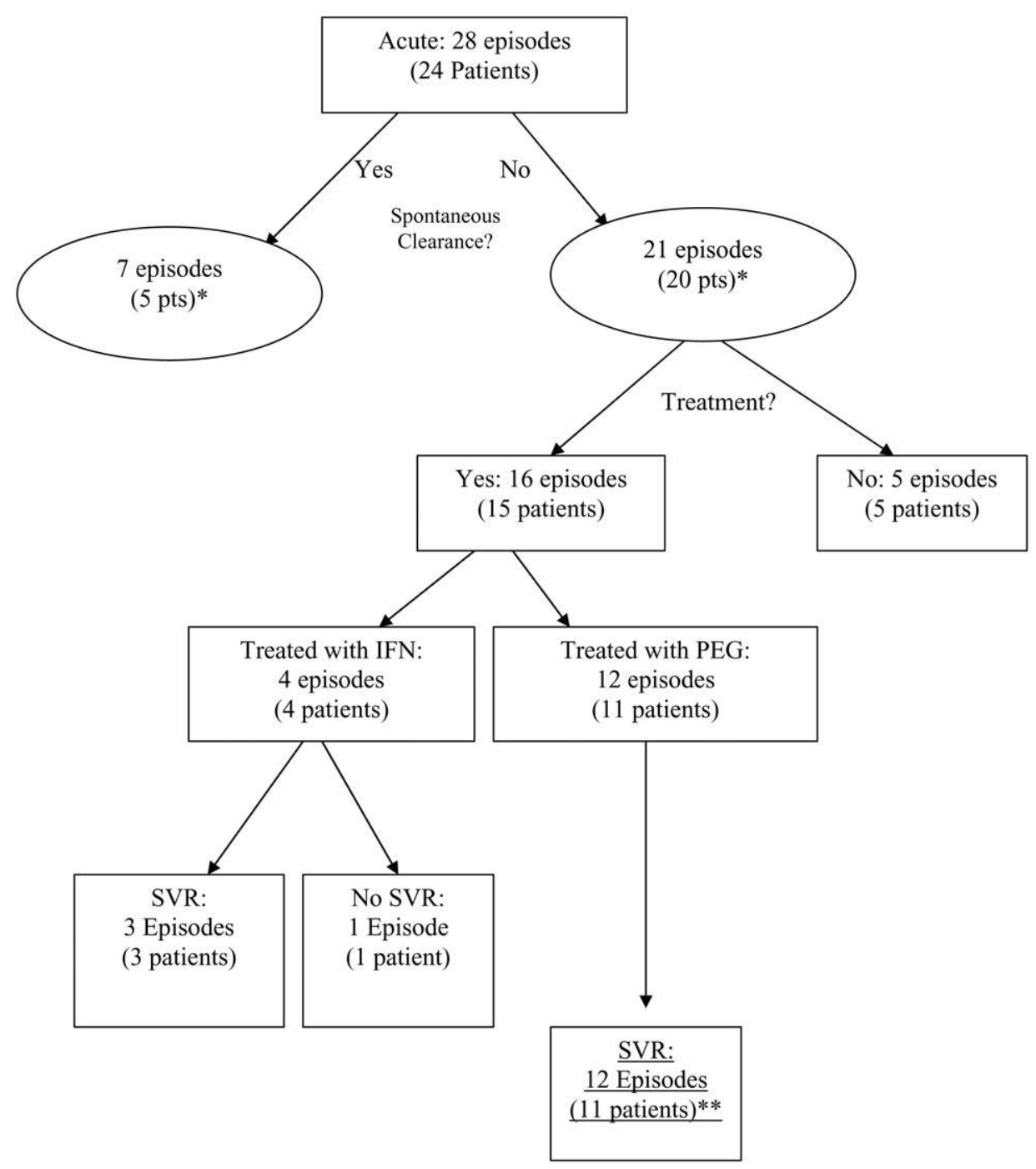

*4 patients experienced two discrete acute infections, 1 of whom had nonclearance with $1^{\text {st }}$ episode and spontaneous clearance with $2^{\text {nd }}$ episode. This patient is therefore counted once as a spontaneous clearance and once as no spontaneous clearance

**1 patient was treated for two discrete acute infections with PEGIFN + RBV and is counted as two SVRs.

Figure 1.

Breakdown of Episodes of Acute HCV infection 


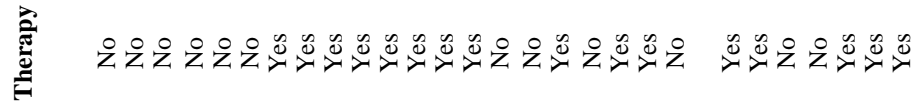

ปัँ

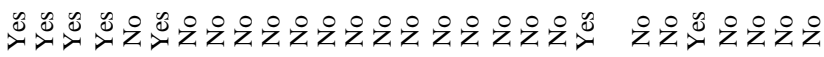

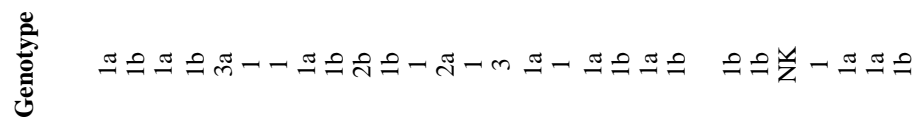

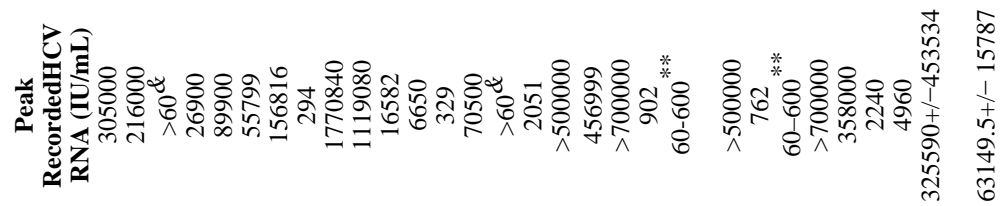

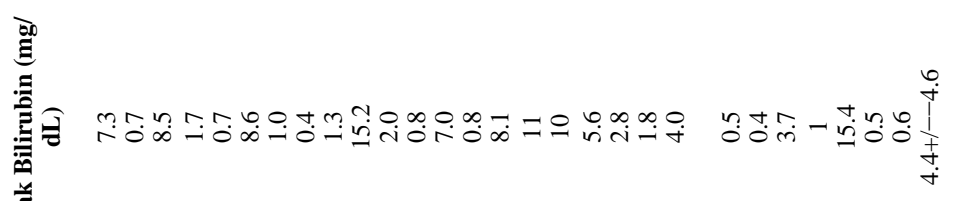

苞

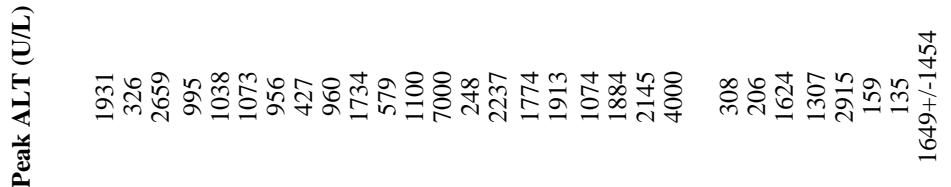

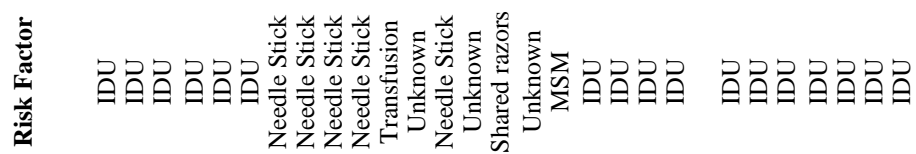


Table 2

Outcomes in patients receiving anti-viral therapy

\begin{tabular}{|c|c|c|c|c|c|c|}
\hline Patient & Treatment Episode & Age & Treatment $* *$ & ETVR & SVR & Treatment Duration (weeks) \\
\hline 5 & 1 & 45 & IFN $\alpha 2 \mathrm{~b} 3 \mathrm{MU}$ tiw/RBV ${ }^{* *}$ & Yes & Yes & 48 \\
\hline 6 & 1 & 40 & IFN $\alpha 2 b$ 3MU tiw/RBV & Yes & Yes & 36 \\
\hline 7 & 1 & 41 & IFN $\alpha 2 \mathrm{~b} 3 \mathrm{MU}$ tiw/RBV & Yes & No & 42 \\
\hline 8 & 1 & 23 & IFN $\alpha 2 \mathrm{~b} 3 \mathrm{MU}$ tiw/RBV & Yes & Yes & 28 \\
\hline 9 & 1 & 15 & $\begin{array}{l}\text { IFN } \alpha 2 b \text { 3MU tiw } \\
\text { Monotherapy }\end{array}$ & Yes & No & 12 \\
\hline 9 & 2 & 16 & PEG Monotherapy ${ }^{*}$ & Yes & Yes & 23 \\
\hline 10 & 1 & 50 & PEG/RBV ${ }^{* * *}$ & Yes & Yes & 24 \\
\hline 11 & 1 & 26 & PEG/RBV & Yes & Yes & 24 \\
\hline 12 & 1 & 63 & PEG/RBV & Yes & Yes & 12 \\
\hline 15 & 1 & 35 & PEG/RBV & Yes & Yes & 24 \\
\hline 17 & 1 & 36 & PEG/RBV & Yes & Yes & 24 \\
\hline 18 & 1 & 23 & PEG/RBV & Yes & Yes & 12 \\
\hline 19 & 1 & 20 & $\mathrm{PEG} / \mathrm{RBV}$ & Yes & Yes & 12 \\
\hline 20 & 1 & 19 & PEG/RBV & Yes & Yes & 24 \\
\hline 23 & 1 & 20 & PEG/RBV & Yes & Yes & 24 \\
\hline 24 & 1 & 23 & PEG/RBV & Yes & Yes & 12 \\
\hline 24 & 2 & 25 & PEG/RBV & Yes & Yes & 12 \\
\hline
\end{tabular}

